\title{
Le syndrome de Prader-Willi : données nouvelles
}

Depuis près de trois ans, $\mathrm{m} / \mathrm{s}$ tient ses lecteurs informés de deux notions qui se chevauchent mais ne se recouvrent pas totalement : les disomies uniparentales dans lesquelles deux chromosomes, homologues viennent d'un seul parent (il s'agit d'une hétérodisomie quand les deux chromosomes sont différents, d'une isodisomie quand un seul chromosome, dupliqué, est présent) ; et l'empreinte, ou sceau, génomique, qui indique que, pour certaines parties du génome, les gènes paternel et maternel ne sont pas équivalents et ne peuvent donc se remplacer $(\mathrm{m} / \mathrm{s}$ $\mathrm{n}^{\circ} 1$, vol. 6 p. 57). Parmi les syndromes où interviennent ces mécanismes il en est deux, symétriques, qui ont fait l'objet de nombreuses recherches ; ils mettent en jeu le chromosome 15, et, plus précisément, la partie proximale de son bras long. Le mieux connu est le syndrome de Prader-Willi (SPW) qui se manifeste par une hypotonie infantile, une petite taille, des dysmorphies modérées et un hypogonadisme. L'autre est le syndrome d'Angelman (SA), marqué surtout par un retard mental avec microcéphalie, des crises épileptiques et des rires incontrôlés. Depuis plusieurs années on sait que dans environ $60 \%$ des cas des délétions sont visibles dans la région $15 \mathrm{q} 12$; elles sont toujours d'origine paternelle dans le SPW, maternelle dans le SA. Les observations les plus frappantes sont celles qui montrent des cas de SPW et de SA dans une même famille. Deux ont été publiées à notre connaissance. Dans la première, déjà décrite dans $m / s\left(n^{\circ} 9\right.$, vol. 7 p. 974), une translocation entre les chromosomes 15 et 22 aboutissait chez certains sujets à une délétion en $15 q 12$; héritée du père, la délétion provoquait un SPW ; de la mère, un SA. L'autre famille [1] décrit deux cousines germaines, victimes d'une translocation 6-15, dont l'une a un SPW avec délétion du 15q12 paternel ; l'autre, atteinte de SA, n'a apparemment pas de délétion, mais ses chromosomes 15 proviennent tous deux du père. Il s'agit, dans ce cas, d'une hétérodisomie. De telles observations ont montré que l'absence d'une région critique en provenance d'un des deux sexes est suffisante pour provoquer un des deux syndromes. Elle n'est pas forcément nécessaire, puisqu'elle manque dans plus d'un tiers des cas. Quelle est, dans ce cas, l'origine de la maladic?

Deux articles récents, sans lever toutes les ambiguïtés, s'efforcent de répondre à cette question. M.J. Mascari, avec huit auteurs américains et anglais [2], a examiné 30 sujets atteints de SPW sans délétions visibles. Chez 8 d'entre eux des délétions moléculaires furent découvertes. Chez $60 \%$ des malades (18 sujets), fut mise en évidence une isodisomie maternelle. Chez les quatre derniers, la distribution parentale semblait correcte. Donc si la présence d'une délétion, ou d'une isodisomie est la règle, il existe des exceptions. L'autre article, du à Gregory $e t$ al. [3] au Canada, aboutit à des conclusions très différentes. Analysant en détail l'ADN de la région incriminée de six malades sans délétion visible, il conclut à des réarrangements moléculaires entraînant une hémizygotie à certains loci avec disparition de certains marqueurs, et ce, toujours dans le chromosome paternel. En revanche, il ne trouve pas d'anomalie dans le chromosome maternel ; il conclut donc à un mécanisme de recombinaisons somatiques et non d'isodisomie uniparentale. Il y a cependant confirmation du phénomène de sceau génomique, puisque la contribution maternelle est présente et non la paternelle. On reste donc dans une position d'attente : dans la grande majorité des cas l'absence de contribution paternelle correcte est confirmée, qu'il y ait ou non disomie uniparentale. Dans quelques cas - et c'est vrai aussi pour le SA - il semble y avoir contribution normale des deux parents. Quelle est la cause ? erreur de diagnostic, cas atypiques, remaniements encore méconnus, ou mécanismes différents encore à découvrir..
Une autre questions restait jusqu'à présent sans réponse certaine. Dans la plupart des cas de disomie uniparentale liée au chromosome 15 , celle-ci ne porte que sur la partie proximale du bras long; qu'en serait-il si l'ensemble des deux 15 provenait d'un même parent? Une observation d'auteurs australiens [4] répond clairement. Un prélèvement de routine de villosités choriales pour âge maternel avancé montra une trisomie 15 dans 50 à $100 \%$ des cellules en cultures. Un examen ultérieur de cellules amniotiques conclut à un caryotype normal 46 XY. Après la naissance, l'hypotonie et l'hypogonadisme firent porter un diagnostic de SPW. L'analyse moléculaire ne montra aucune délétion, mais la totalité des chromosomes 15 provenait de la mère. Il s'agissait d'une hétérodisomie maternelle. Ce cas donc dérivait d'une trisomie 15 , un des chromosomes, celui du père, ayant secondairement disparu. Ce cas montre pour la première fois comment s'est produite la disomie uniparentale. Mais il recèle plusieurs éléments d'intérêts supplémentaire : le tableau clinique est celui d'un SPW non particulièrement sévère ; par conséquent seule la zone q12 serait soumise à empreinte parentale génomique, et l'absence de contribution paternelle dans le reste du 15 n'entraînerait pas d'autre symptôme. Débordant le cadre du chromosome 15 , on peut remarquer que la perte secondaire d'un chromosome trisomique est un mécanisme salvateur, même si, par malchance, les deux qui restent proviennent du même parent. On peut noter enfin qu'une mosaïque placentaire disparaissant par la suite peut indiquer assez souvent un mécanisme correcteur d'une trisomie. Il serait intéressant, dans de tels cas, de rechercher la possibilité d'une contribution uniparentale pour les deux chromosomes qui subsistent. On pourrait ainsi savoir quels chromosomes sont porteurs de sceau génomique parental.

J.C.D. 
1. Smeets DFCM, Hamel BCJ, Nelen MR et al. Prader-Willi syndrome and Angelman syndrome in cousins from a family with a translocation between chromosomes 6 and 15. N Engl J Med 1992 ; 326 : 807-11.

2. Mascari MJ, Gottlieb W, Rogan PK et al. The frequency of uniparental disomy in Prader-Willi syndrome. New Engl J Med 1992 ; 326 : 1599-60.

3. Gregory CA, Schwartz J, Kirkilionis AJ, Rudd N, Hamerton JL. Somatic recombination rather than uniparental disomy suggested as another mechanism by which genetic imprinting may play a role in the etiology of Prader-Willi syndrome. Hum Genet 1991 ; 88 : 42-8

4. Purvis-Smith SG, Saville T, Manass S et al. Uniparental disomy 15 resulting from " correction" of an initial trisomy 15. Am J Hum Genet 1992 ; 50 : 1348-50.

\section{Note ajoutée aux épreuves}

Un travail tout récent d'un groupe de Gainsville (FL, USA) [5], a identifié un ADNc localisé en 15q11-q13, appelé DN 34 , qui présente une différence de méthylation entre les deux allèles parentaux. Les auteurs pensent que le gène correspondant est un candidat pour SWP et SA.

5. Driscoll DJ, Waters MF, William CA et al. A DNA methylation imprint, determined by the sex of the parent, distinguishes the Angelman and Prader-Willi syndromes. Genomirs 1992, 13 : 917-24.

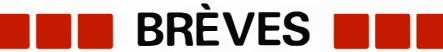

Le syndrome de Lowe : une anomalie du système des inositolphosphates? Le syndrome oculocérébro-rénal de Lowe est une maladie récessive liée à l'X. Ses manifestations principales sont une cataracte congénitale, un retard mental et un dysfonctionnement tubulaire rénal. La localisation de son gène en Xq25-26 par liaison génétique a été confirmée par deux cas féminins avec translocation X-autosome. Une équipe américano-canadienne [1] a utilisé 2 chromosomes artificiels de levure, qui enjambent le point de cassure d'une de ces translocations, comme sondes pour cribler une banque rénale humaine d'ADNc. O. Attree, un jeune Français en stage post-doctoral aux États-Unis, et ses collègues ont réussi à identifier un ADNc capable de coder pour une protéine de 968 acides aminés et de masse 112000 . Il reconnaît un messager de 5,8 kb présent dans de nombreux tissus. Ce transcrit est absent chez les deux femmes victimes de translocations, et chez 8 des 15 hommes atteints examinés. Un 9e malade avait un transcrit trop grand, de $6,5 \mathrm{~kb}$. Dans aucun cas l'ADN étudié par la méthode de Southern ne paraissait anormal, indiquant qu'il ne devait pas s'agir de délétions ou de remaniements importants. Une recherche dans les banques de données décela une ressemblance (53\% d'identité) avec une inositol polyphosphate 5-phosphatase de plaquettes humaines de $75000 \mathrm{Da}$ [2]. Les différences étant réparties tout au long de la molécule, il ne s'agit cependant pas du même gène. On s'oriente actuellement vers d'autres inositol phosphatases isolées du cerveau [3, 4]. Si ces résultats sont confirmés - et cette restriction est importante car aucune preuve n'existe quant à la fonction du gène nouvellement découvert - ils s'avéreront d'une importance extrême. Ce serait en effet la première maladie génétique humaine affectant les voies métaboliques des inositol-phosphates. On pourrait peut-être en outre comprendre comment l'inactivation d'une seule enzyme, placée sur une voie générale, peut engendrer des symptômes à la fois aussi pléiotropes et aussi précis.

[1. Attree O, et al. Nature $1992 ; 358$ : 239-42.

[2. Ross TS, et al. J Biol Chern 1991 ; 266 : 20283-9.]

[3. Hansen CA, et al. J Biol Chem 1987 ; 262 : 17319-26.]

[4. Erneux C, et al. Eur J Biochem $1989 ; 181: 317-22$.] 